\title{
Influence of L-Sorbose on the Growth and Morphology of Neurospora crassa
}

\author{
By A. P. J. TRINCI AND ANNETTE COLLINGE \\ Microbiology Department, Queen Elizabeth College, Campden Hill, \\ London, $W .8$
}

(Received 22 March 1973)

SUMMARY

The effects of L-sorbose on the diameter, compartment length, wall thickness and fine structure of hyphae from colonies and batch cultures of Neurospora crassa (spco I and spco 9) were studied. The specific growth rates and yields of the strains in batch culture and their germ-tube specific growth rates were at most reduced by only I $8 \%$ when $2 \%$ L-sorbose was added to Vogel's medium containing I $\%$ sucrose.

The addition of L-sorbose to solid medium induced both strains to branch profusely and resulted in the formation of dense, slow growing colonies (about $90 \%$ reduction in radial growth rate). This reduction in the radial growth rate of spco 9 colonies could be largely correlated with a reduction (from $4.0 \mathrm{~mm}$ to $0.5 \mathrm{~mm}$ ) in the width of the peripheral growth zone of colonies grown on media containing L-sorbose. Plugging of the septal pores and autolysis of hyphae of spco 9 occurred closer to the colony's periphery when L-sorbose was present in the medium. L-Sorbose reduced the number and size of the vesicles found at the tips of hyphae of spco 9 .

\section{INTRODUCTION}

The addition of L-sorbose to solid media containing another carbon source induces many fungi to form dense colonies which extend radially at much slower rates than control colonies (Tatum, Barratt \& Cutter, 1949; Lysek \& Esser, 1971; Moore \& Stewart, 1972). Similar effects on the morphology and growth rate of fungal colonies are induced by cellobiose, 2-deoxy-D-glucose, galactose and D-glucosamine (Wilson \& Neiderpruem, I967; Lysek \& Esser, I97I; Moore \& Stewart, 1972). The effects of these compounds can often be partially reversed by increasing the concentration of the normal carbon source (glucose or fructose) in the medium (Lysek \& Esser, I97I; Moore \& Stewart, I972).

The radial growth rate $\left(K_{r}\right)$ of a fungal colony may be defined (Trinci, 197I) by the equation

$$
K_{r}=w \alpha,
$$

where $\alpha=$ the mould's specific growth rate and $w=$ the width of the peripheral zone of the colony which contributes to its radial expansion. The radial growth rate of a colony will thus be altered by factors which change either of the two functions shown in equation (I).

The present study was undertaken to compare the effects of L-sorbose on the growth rate and morphology of Neurospora crassa cultured on solid media and in batch culture. 


\section{METHODS}

Organisms. Neurospora crassa spco I and spco 9 were kindly supplied by the Fungal Genetics Stock Centre, Humboldt State College Foundation, Arcata, California; spco = spreading colonial mutant.

Media. The strains were grown on Vogel's (1956) minimal medium with I \% (w/v) sucrose as the normal carbon source; sucrose is the limiting nutrient in this medium. L-Sorbose was prepared as a 5 or $10 \%(w / v)$ aqueous solution, sterilized at $10 \mathrm{lb} / \mathrm{in}^{2}$ for I 5 min and added to the other ingredients (Trinci, 1973 $a$ ) aseptically.

The medium was usually gelled with Oxoid (London, S.E.I) Ion Agar no. 2 (final concentration $\mathrm{I} \cdot 5 \%$ but media were also gelled with $\mathrm{I} \cdot 5 \%$ (final concentration) pure agarose (Koch-Light Laboratories, Colnbrook, Buckinghamshire) or HS-40 colloidal silica (Dupont Co. Ltd, London, E.C. 4). Silica-gel plates were prepared by adding sterile ( $5 \mathrm{~min}$ at $10 \mathrm{lb} /$ in $^{2}$ ) HS-40 colloidal silica to an equal volume of the remaining ingredients; the final medium contained $20 \%(\mathrm{w} / \mathrm{v})$ silica and had a $\mathrm{pH}$ of 5.5 .

Colonies were grown in $9 \mathrm{~cm}$ plastic Petri dishes containing $20 \mathrm{ml}$ of medium. Plate cultures were inoculated and incubated at $25^{\circ} \mathrm{C}$ as described previously (Trinci, I973a).

Measurement of the peripheral growth zone of colonies and germ-tube specific growth rate

The peripheral growth zones of the colonies were determined by time-lapse photography (Trinci, I973a) by means of the method of Clutterbuck \& Roper (I966) and Lhoas (I968). The growth of germ tubes on solid media was determined as described previously (Trinci, I973a).

\section{Batch cultures}

Batch cultures were grown at $25^{\circ} \mathrm{C}$ in $250 \mathrm{ml}$ flasks containing $25 \mathrm{ml}$ of medium. The cultures were inoculated with conidia, shaken at $200 \mathrm{rev} . / \mathrm{min}$, harvested and weighed as described previously (Trinci, 1973a). The organism yield $(Y)$ was calculated from the following equation,

\section{Light microscopy}

$$
Y=\frac{\text { maximum organism dry } \mathrm{wt} / \mathrm{ml} \text { of culture } \times \mathrm{IOO}}{\mathrm{wt} \text { of sucrose } / \mathrm{ml} \text { of medium }} .
$$

Measurements of the diam. and compartment length of leading hyphae were made on mature colonies which had been fixed in $20 \%(\mathrm{v} / \mathrm{v})$ aqueous glutaraldehyde at $4{ }^{\circ} \mathrm{C}$ (Trinci, I $973 a$ ). Hyphae from batch cultures were sampled $\mathrm{I} 6$ to $\mathrm{I} 9 \mathrm{~h}$ after inoculation and fixed in equal volumes of glutaraldehyde or FAA (Righelato, Trinci, Pirt \& Peat, I968). The mean hyphal diameters of these samples were determined.

The frequency of hyphal branching was determined by calculating the hyphal growth unit (Caldwell \& Trinci, 1973; Trinci, I973b). The growth unit (HGU) of a hypha is defined by the equation,

$$
\text { HGU }=\frac{\text { total length of a hypha }(\mu \mathrm{m})}{\text { number of tips }} .
$$

The growth units of hyphae which had three to six tips were determined. These hyphae were measured after $\mathrm{I} 6$ to $\mathrm{I} 8 \mathrm{~h}$ growth at $25^{\circ} \mathrm{C}$ on plate cultures overlaid with cellophane (Trinci, $\mathrm{I} 973 b$ ); the cellophane ensured that growth occurred in a single plane.

\section{Electron microscopy}

The methods used for electron microscopy have been described previously (Trinci \& Collinge, 1973). The \% of plugged septal pores was in each case calculated from observa- 


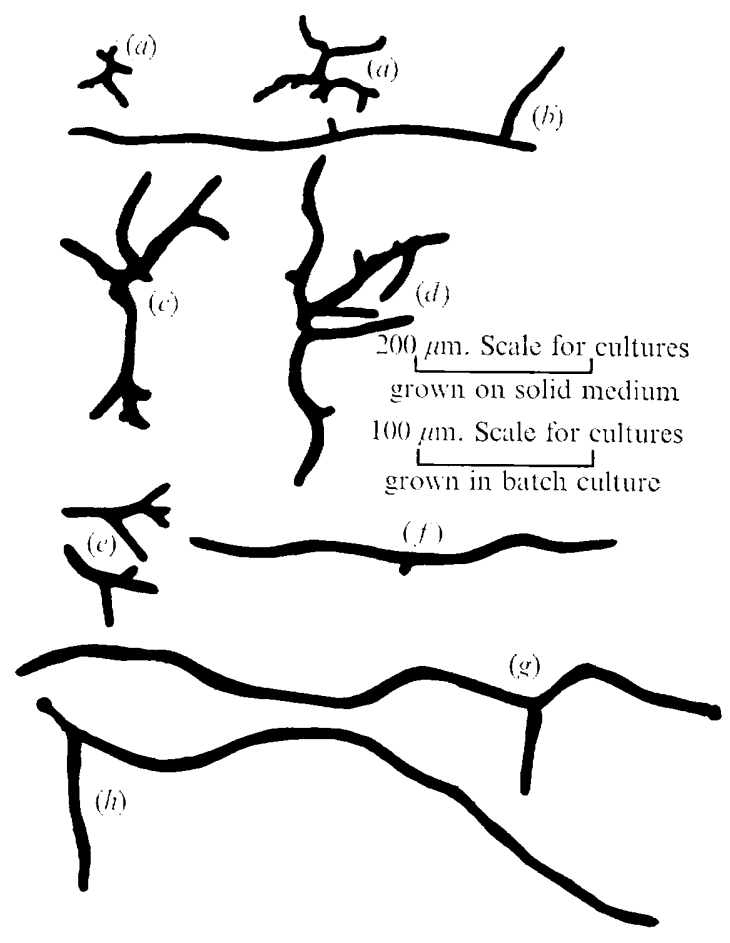

Fig. I. Hyphae of Neurospora spco I and spco 9 grown in the presence and absence of $2 \% \mathrm{~L}$-sorbose in liquid and solid culture. Tracings made from photographs.

$$
\begin{array}{ccc}
\text { Strain } & 2 \% \text { L-sorbose } \\
\text { in medium } & \begin{array}{c}
\text { Solid or batch } \\
\text { culture }
\end{array}
\end{array}
$$

$\begin{array}{lll}\text { (a) } \text { spco I } & \text { Present } & \text { Solid } \\ (b) \text { spco I } & \text { Absent } & \text { Solid } \\ (c) \text { spco I } & \text { Present } & \text { Batch } \\ (d) \text { spco I } & \text { Absent } & \text { Batch } \\ (e) \text { spco } 9 & \text { Present } & \text { Solid } \\ (f) \text { spco } 9 & \text { Absent } & \text { Solid } \\ (g) \text { spco } 9 & \text { Absent } & \text { Batch } \\ (h) \text { spco } 9 & \text { Present } & \text { Batch }\end{array}$

tions made on 5 to I 26 septa sampled at various distances from the colony's periphery. Measurements of wall thickness were only made on hyphae which had a normal cytology; intrahyphal hyphae were excluded from these determinations which in each case was the mean of 100 measurements made on at least 20 different hyphae. The determinations of vesicle diam. were the mean of 100 measurements.

\section{RESULTS}

Influence of L-sorbose on the morphology of hyphae grown in batch culture

L-Sorbose had no effect on the macroscopic appearance of batch cultures of spco 9; cultures were filamentous even in the presence of L-sorbose. However, L-sorbose frequently increased the concentration of pellets in batch cultures of spco $I$.

L-Sorbose had no significant effect on the diameter (Table I $a$ ), fine structure or length of the growth unit (Table 2) of hyphae of spco I and spco 9 grown in batch culture (Fig. I). 
Table I. Influence of L-sorbose on hyphal diameter, wall thickness and compartment length of hyphae grown in batch culture and on solid medium

(a) Hyphae grown in submerged culture

\begin{tabular}{|c|c|c|c|c|}
\hline \multirow[b]{2}{*}{ Strain } & \multicolumn{2}{|c|}{ Mean wall thickness (nm) } & \multicolumn{2}{|c|}{ Mean hyphal diam. $(\mu \mathrm{m})$} \\
\hline & $\begin{array}{l}\text { Vogel's } \\
\text { medium }\end{array}$ & $\begin{array}{c}\text { Vogel's medium } \\
+2 \% \\
\text { L-sorbose }\end{array}$ & $\begin{array}{l}\text { Vogel's } \\
\text { medium }\end{array}$ & $\begin{array}{c}\text { Vogel's medium } \\
+2 \% \\
\text { L-sorbose }\end{array}$ \\
\hline & $102 \pm 26^{*}$ & $157 \pm 43^{*}$ & $9 \cdot 0 \pm \mathrm{I} \cdot 8$ & $8 \cdot 2 \pm \mathrm{I} \cdot 4$ \\
\hline & $66 \pm 27$ & $73 \pm 20$ & $3 \cdot 3 \pm 0 \cdot 6$ & $3 \cdot 3 \pm 0.3$ \\
\hline
\end{tabular}

(b) Hyphae from colonies grown on solid medium

\begin{tabular}{|c|c|c|c|c|}
\hline \multirow[b]{2}{*}{ Strain } & \multicolumn{2}{|c|}{$\begin{array}{l}\text { Mean wall thickness (nm) of } \\
\text { hyphae from mature colonies }\end{array}$} & \multicolumn{2}{|c|}{$\begin{array}{l}\text { Mean hyphal diameter of } \\
\text { young mycelia less than I } \mathrm{mm} \\
\text { in total length }(\mu \mathrm{m})\end{array}$} \\
\hline & $\begin{array}{l}\text { Vogel's } \\
\text { medium }\end{array}$ & $\begin{array}{l}\text { Vogel's medium } \\
+2 \% \\
\text { L-sorbose }\end{array}$ & $\begin{array}{l}\text { Vogel's } \\
\text { medium }\end{array}$ & $\begin{array}{l}\text { Vogel's medium } \\
+2 \% \\
\text { L-sorbose }\end{array}$ \\
\hline $\begin{array}{l}\text { pco I } \\
\text { pco } 9\end{array}$ & $\begin{array}{l}84 \pm 23 \dagger \\
74 \pm 23 \dagger\end{array}$ & $\begin{array}{l}179 \pm 96 \dagger \\
106 \pm 34 \S\end{array}$ & $\begin{array}{l}9.5 \pm 1 \cdot 2 \\
7 \cdot 7 \pm 0.9\end{array}$ & $\begin{array}{r}10 \cdot 3 \pm \mathrm{I} \cdot 4 \\
8 \cdot 3 \pm 0 \cdot 8\end{array}$ \\
\hline
\end{tabular}

(c) Leading hyphae at the margin of mature colonies of spco 9 grown on solid medium

\begin{tabular}{|c|c|c|c|}
\hline Parameter measured $(\mu \mathrm{m})$ & $\begin{array}{l}\text { Vogel's } \\
\text { medium }\end{array}$ & $\begin{array}{c}\text { Vogel's medium } \\
+1 \% \\
\text { L-sorbose }\end{array}$ & $\begin{array}{c}\text { Vogel's medium } \\
+2 \% \\
\text { L-sorbose }\end{array}$ \\
\hline mpartment & 38 & $120 \pm 33$ & $72 \pm 22$ \\
\hline (ii) Second compartment & $164 \pm 66$ & $38 \pm 14$ & $32 \pm 7$ \\
\hline (iii) Third compartment & $149 \pm 77$ & $46 \pm 16$ & $29 \pm 5$ \\
\hline (iv) Diameter & $8 \cdot 9 \pm I \cdot 0$ & $3 \cdot 2 \pm 0.5$ & $2 \cdot 4 \pm 0.8$ \\
\hline
\end{tabular}

\footnotetext{
* Measurements made on healthy hyphae from the centre of pellets which had a diameter of $\mathrm{I}$ to $2 \mathrm{~mm}$.

$\uparrow$ Measurements made on hyphae in o to $3 \mathrm{~mm}$ region from colony's margin.

$\$$ Measurements made on hyphae in 2 to $9 \mathrm{~mm}$ region from colony's margin.

$\S$ Measurements made on hyphae in 0 to $5 \mathrm{~mm}$ region from colony's margin.
}

\section{Influence of L-sorbose on the morphology of hyphae grown on solid media}

L-Sorbose had no effect (Table I $b$ ) on the hyphal diameter of young mycelia ( 16 to $18 \mathrm{~h}$ after inoculation) formed on solid media. Branch initiation was increased (Fig. I) when spco I and spco 9 were grown on solid media containing L-sorbose and consequently there was a considerable reduction in the length of the growth units of these strains (Table 2). This decrease in the length of the hyphal growth unit was observed even when the hyphae were in direct contact with the medium (i.e. in the absence of cellophane). As a direct result of the increase in branch initiation very dense colonies were formed on media containing L-sorbose.

The length of apical and intercalary compartments and the diameter of leading hyphae at the fringe of mature colonies of spco 9 were all reduced by L-sorbose (Table I $c$ ). The mean wall thickness of hyphae sampled from mature colonies of spco I and spco 9 were increased by L-sorbose (Table $\mathrm{I} b$ ); the hyphae in these samples were not necessarily of the same age. Intrahyphal hyphae (Fig. 2) were more abundant in colonies of spco I and spco 9 grown on L-sorbose than in control colonies. Intrahyphal hyphae and hyphae which were clearly disorganized cytologically (i.e. they had the appearance of autolysing hyphae, 
Growth of N. crassa

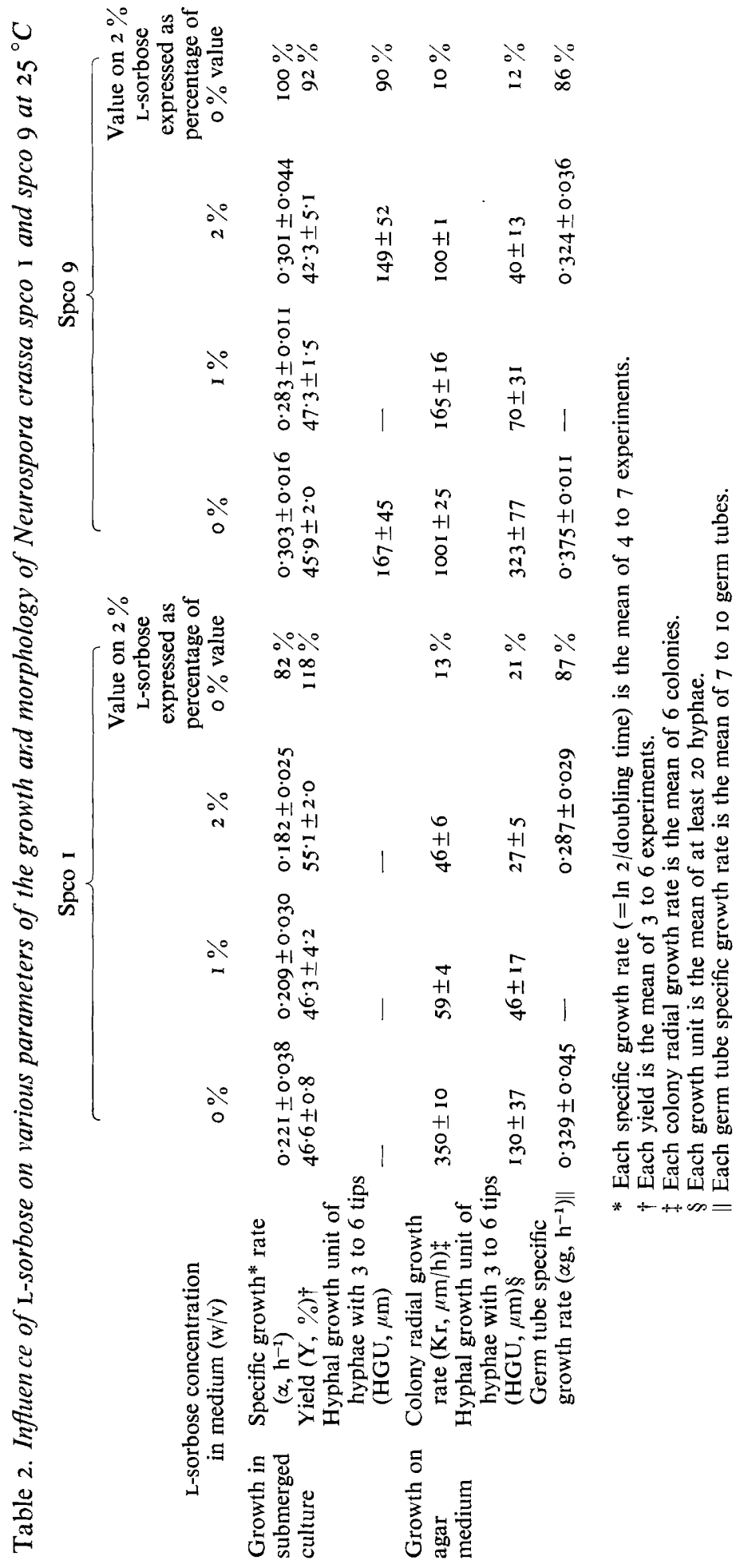




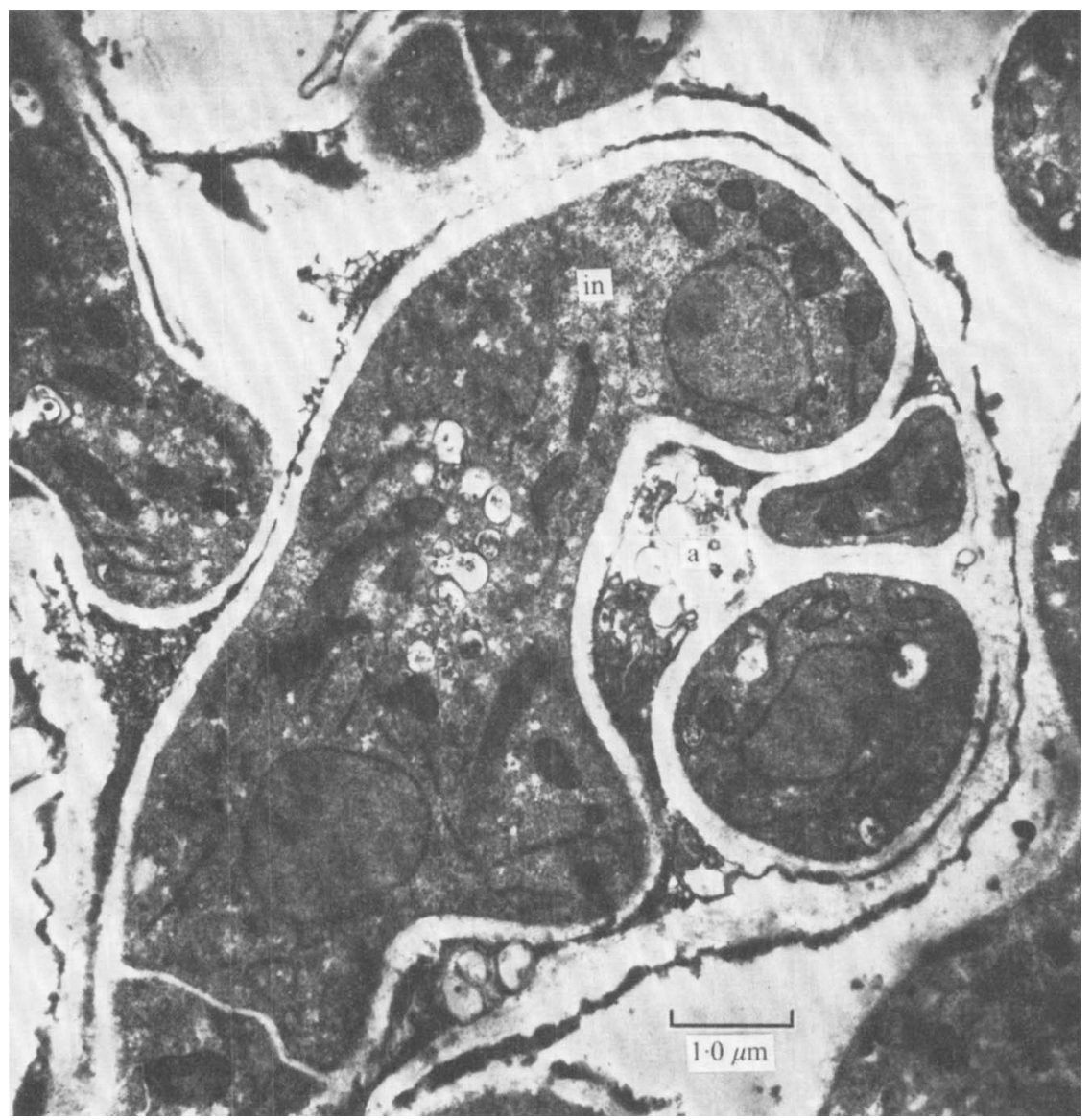

Fig. 2. Intra-hyphal hypha of Neurospora crassa spco 9 in 2 to $2.5 \mathrm{~mm}$ region from the margin of a colony grown on $2 \% \mathrm{~L}$-sorbose. a, Autolysing protoplasm; in, intra-hyphal hypha.

Trinci \& Righelato, I971) were frequently observed in the 0.5 to $\mathrm{I} \mathrm{mm}$ region from the periphery of colonies of spco 9 grown on $2 \%$ L-sorbose (Fig. 3). Such hyphae were only rarely observed in control colonies of spco 9 and were not found in the peripheral $8 \mathrm{~mm}$ of the colony.

The tips of leading hyphae of colonies of spco 9 grown on media containing $2 \%$ L-sorbose contained far fewer vesicles (Fig. 6) than the tips of leading hyphae of control colonies (Fig. 5). Further, the mean diam. of the former vesicles was significantly less than that of the latter $(59 \pm 17 \mathrm{~nm}$ as compared with $\mathrm{I} 23 \pm \mathrm{I} 9 \mathrm{~nm})$. The mean diameter of the vesicles found at the tips of control spco I hyphae was $62 \pm 2$ I nm (Trinci \& Collinge, I973); there thus appears to be a relationship between vesicle size and the rate of hyphal extension. It is generally accepted that these vesicles contain cell wall precursors which are being transported to the extending primary wall at the hyphal tip.

Plugged septal pores (Fig. 4) were observed much closer to the margin of colonies of spco 9 when L-sorbose was incorporated in the medium (Fig. 7).

Since L-sorbose apparently increased the frequency of branch formation of hyphae grown on solid but not in liquid media (Table 2, Fig. I) it was considered possible that the morpho- 

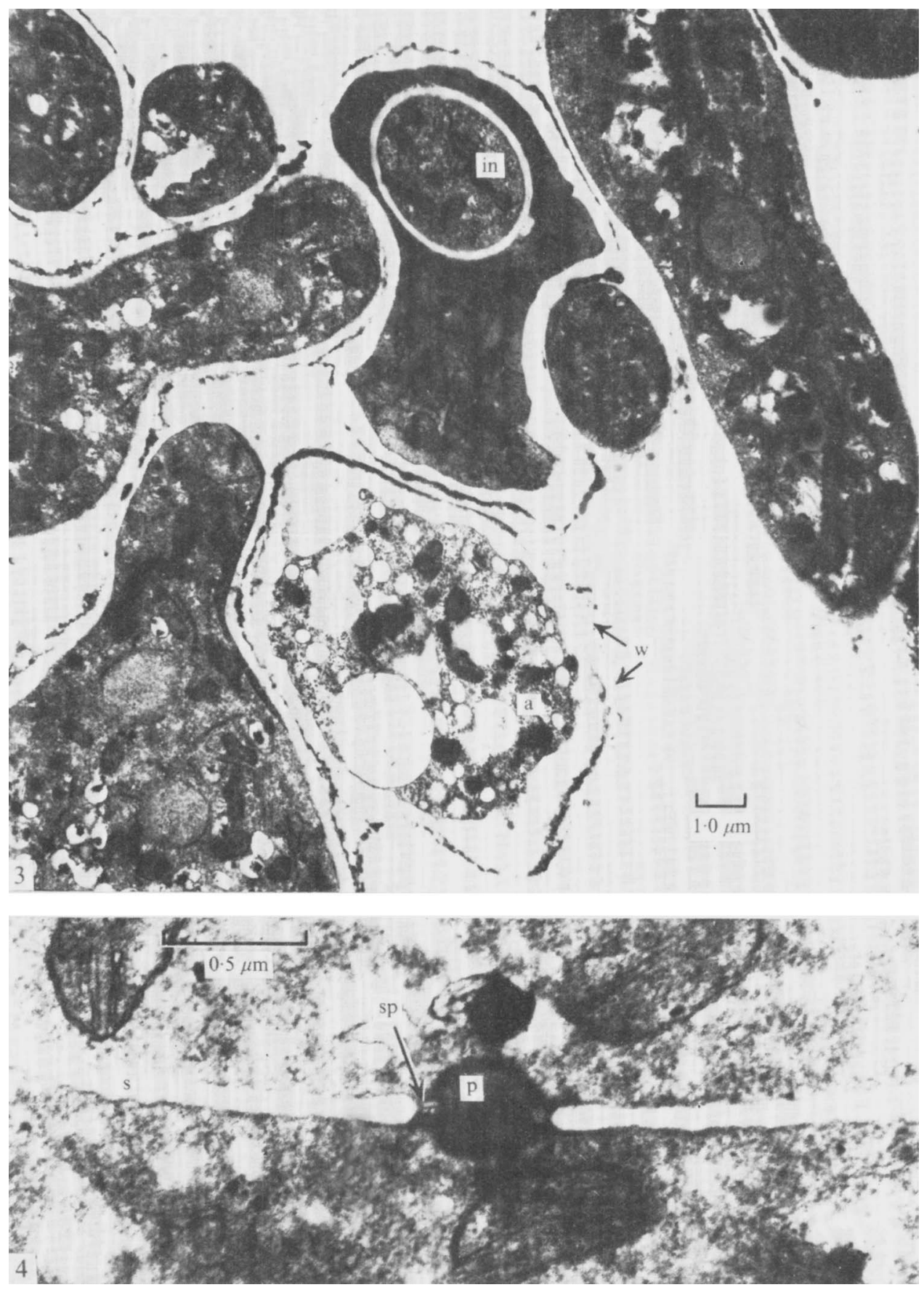

Fig. 3. Autolysing hypha of Neurospora crassa spco 9 in 2 to $2.5 \mathrm{~mm}$ region from the margin of a colony grown on $2 \% \mathrm{~L}$-sorbose. w, Hyphal wall; a, autolysing protoplasm; in, intra-hyphal hypha. Fig. 4. A plugged septal pore of a hypha of Neurospora crassa spco 9 in the 2.5 to $3.0 \mathrm{~mm}$ region from the colony's margin. s, Septal wall; sp, septal projections; $p$, septal plug; $2 \%$ L-sorbose. 

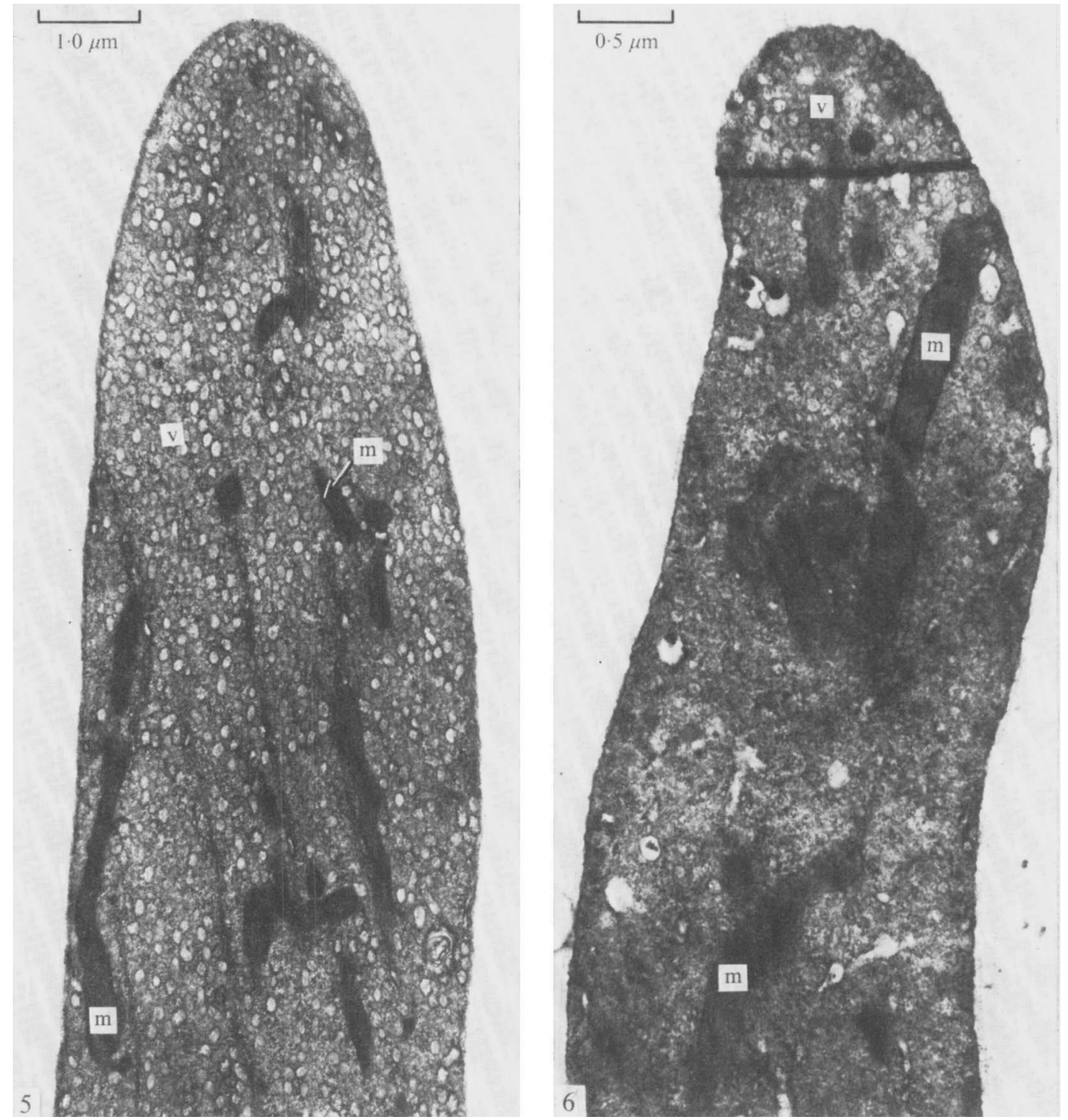

Fig. 5. Tip of a leading hypha of a colony of spco 9 grown on medium lacking L-sorbose. v, Vesicles; $\mathrm{m}$, mitochondrion.

Fig. 6. Tip of a leading hypha of a colony of spco 9 grown on medium containing $2 \%$ L-sorbose. $\mathrm{v}$, Vesicles; $\mathrm{m}$, mitochondrion.

genetic effect of L-sorbose might in some way be related to the composition of the agar used to gel the medium. However, L-sorbose had similar effects on hyphal morphology and colony radial growth rate (Table 3) when agarose or colloidal silica were used to gel the media; dense, slow growing colonies which had narrow hyphae and short hyphal compartments were formed when media containing L-sorbose was gelled with any of the substances shown in Table 3. 
Table 3. Influence of L-sorbose on the radial growth rate of colonies of spco 1 and spco 9 grown at $25^{\circ} \mathrm{C}$ on media gelled with various substances

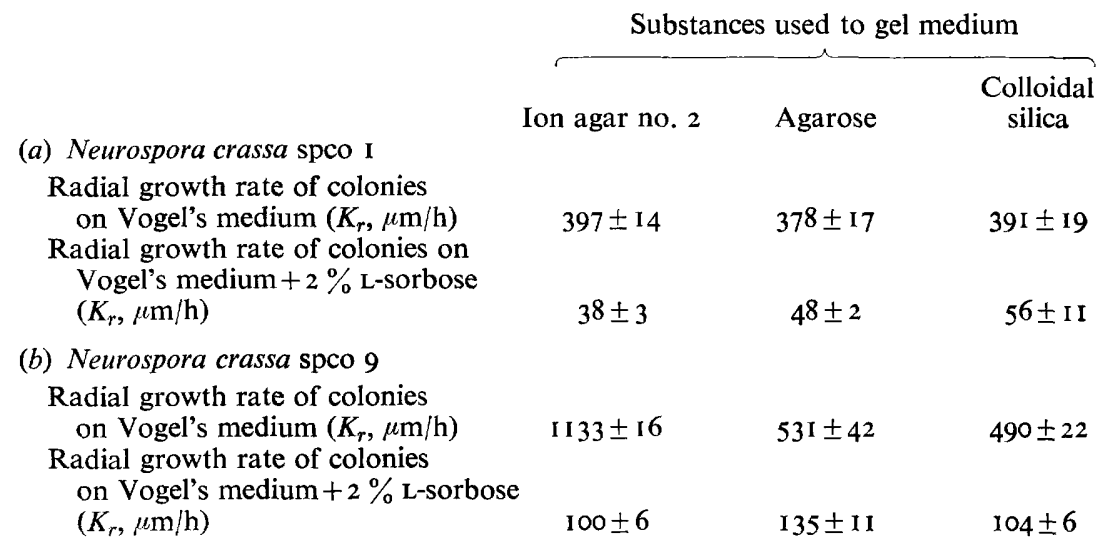

Effect of L-sorbose on the lag, specific growth rate and yield of Neurospora crassa spco I and spco 9 in batch culture

Growth of Neurospora crassa spco I and spco 9 did not occur when L-sorbose was the sole carbon source in the medium (Io to I4 days of incubation). However, spco I, unlike spco 9, did metabolize L-sorbose when the medium contained sucrose in addition to Lsorbose (Fig. 8). It is well established that some fungi will metabolize certain carbon sources only after a period of growth on another carbon source (Cochrane, 1958).

L-Sorbose increased the duration of the lag phases of cultures of spco I and spco 9 (Fig. 9) by about 2 and $3 \mathrm{~h}$ respectively.

Although $2 \%$ L-sorbose had no effect on the specific growth rate of batch cultures of spco 9 it reduced the specific growth rate of batch cultures of spco I by about I $8 \%$ (Table 2 ). However, when spco I was grown in flasks containing $20 \mathrm{ml}$ of medium plus $80 \mathrm{ml}$ of water (concentrations of L-sorbose and sucrose of $0.2 \%$ ) the mould's specific growth rate was $0.243 \pm 0.02 \mathrm{~h}^{-1}$ in the presence of L-sorbose and $0.256 \pm 0.04 \mathrm{~h}^{-1}$ in its absence; some Neurospora crassa strains grow in a more filamentous manner when there is a large vol. of medium/flask and as a result faster specific growth rates are obtained (Trinci, 1973a).

The yield of batch cultures of spco 9 was only slightly decreased by $2 \%$ L-sorbose whilst that of spco I was unaffected by $1 \%$ L-sorbose (Table 2 ). The increased yield of spco I in the presence of $2 \% \mathrm{~L}$-sorbose was almost certainly due to the utilization of this sugar as a carbon source. Sorbose was utilized for growth despite the fact that cultures were harvested at or near the time at which it was anticipated that the sucrose would have only just been exhausted.

Effect of L-sorbose on germ-tube specific growth rate and colony radial growth rate on solid media

L-Sorbose caused an increase of $4 \mathrm{~h}$ in the duration of the lag phase which preceded the emergence of germ tubes from conidia of spco I (Fig. I0). The specific growth rates of germ tubes of spco I and spco 9 were only reduced by $\mathrm{I} 3$ and $14 \%$ respectively when the medium contained $2 \%$ L-sorbose (Table 2 ). However, even $\mathrm{I} \%$ L-sorbose caused a considerable reduction in the radial growth rates of colonies of spco I and spco 9 (Table 2); colonies grown on L-sorbose were denser and had a more irregular margin than control colonies. 


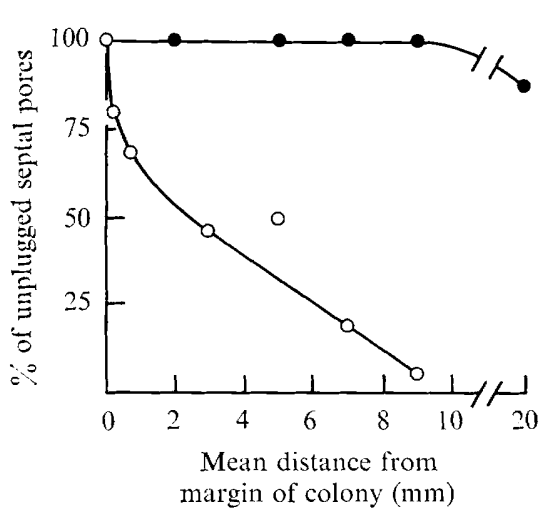

Fig. 7

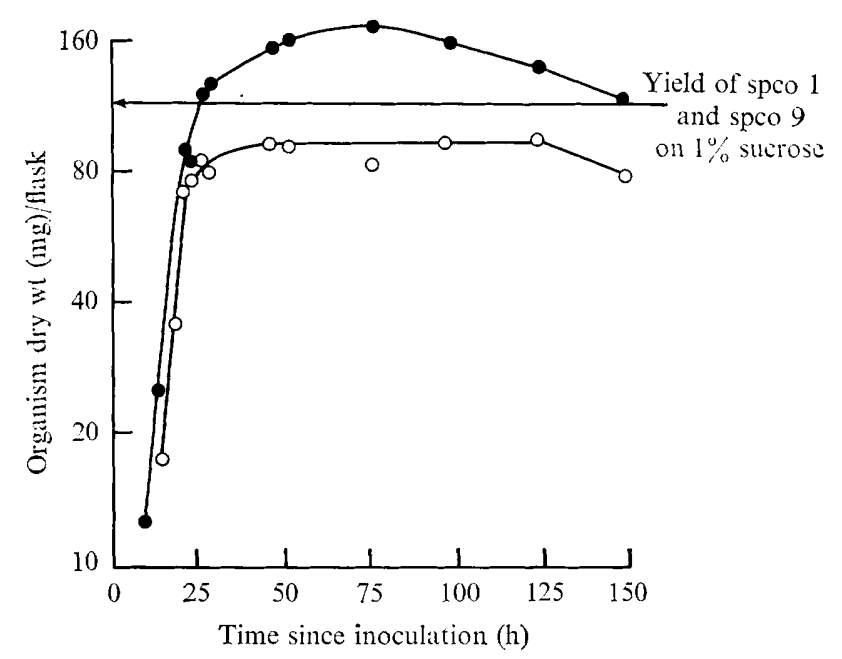

Fig. 8

Fig. 7. Variation in the $\%$ of septal pores which are plugged with distance from the margin of colonies of Neurospora crassa spco 9 grown in the presence $(\bigcirc)$ and absence $(O)$ of $2 \%$ L-sorbose. Fig. 8. Growth of Neurospora crassa spco I (O) and spco $9(\bigcirc)$ at $25{ }^{\circ} \mathrm{C}$ in batch cultures containing I \% sucrose and $2 \%$ L-sorbose.

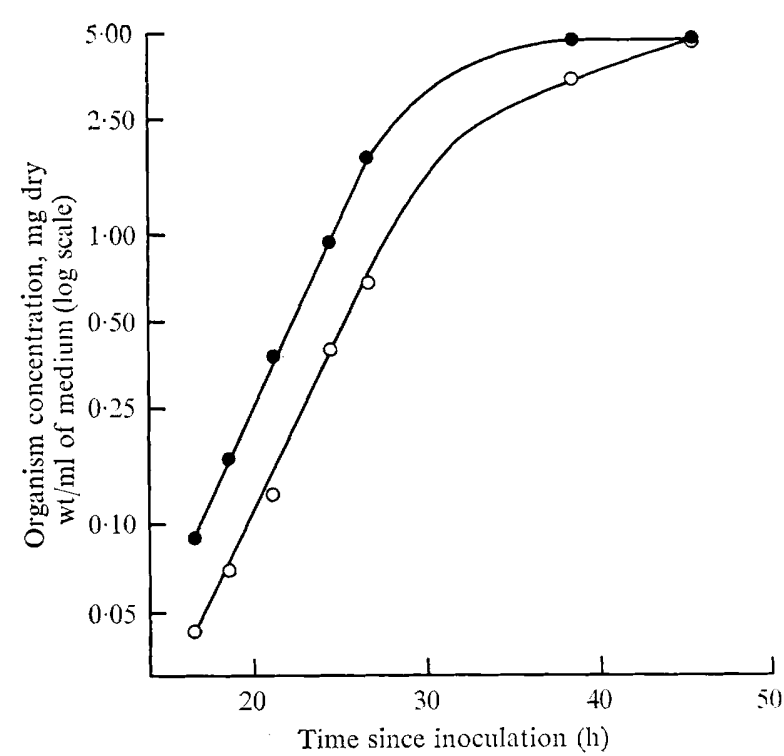

Fig. 9

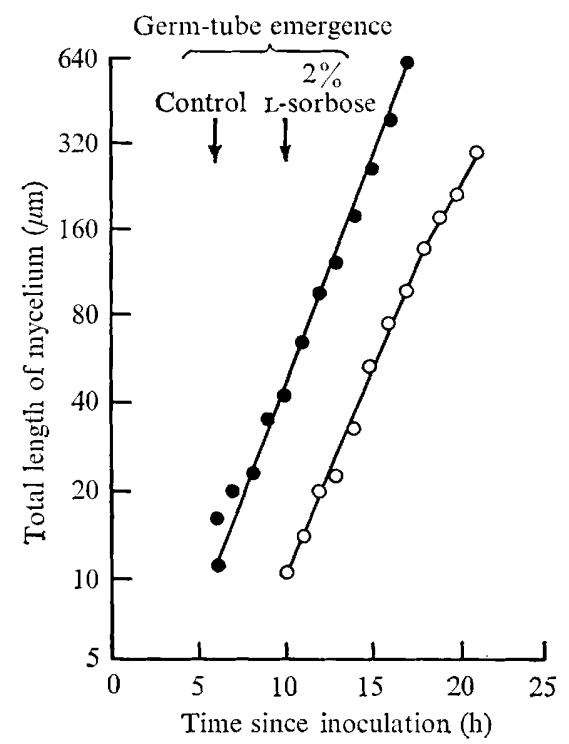

Fig. I0

Fig. 9. Growth of Neurospora crassa spco 9 at $25{ }^{\circ} \mathrm{C}$ in the presence $(O)$ and absence (-) of $2 \%$ L-sorbose.

Fig. 10. Growth of the germ tubes of Neurospora crassa spco I at $25{ }^{\circ} \mathrm{C}$ on solid media containing (O) and lacking (O) $2 \%$ L-sorbose. 


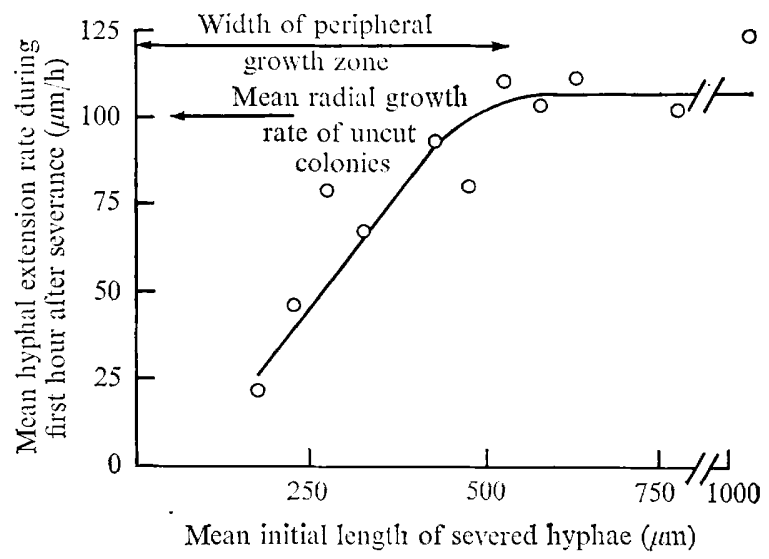

Fig. I I. Extension rates of severed, leading hyphae of colonies of Neurospora crassa spco 9 grown on $2 \%$ L-sorbose. The extension rates were measured during the first hour after the hyphae had been severed. The hyphal extension rates are the mean of groups of hyphae which differed in length by $50 \mu \mathrm{m}$.

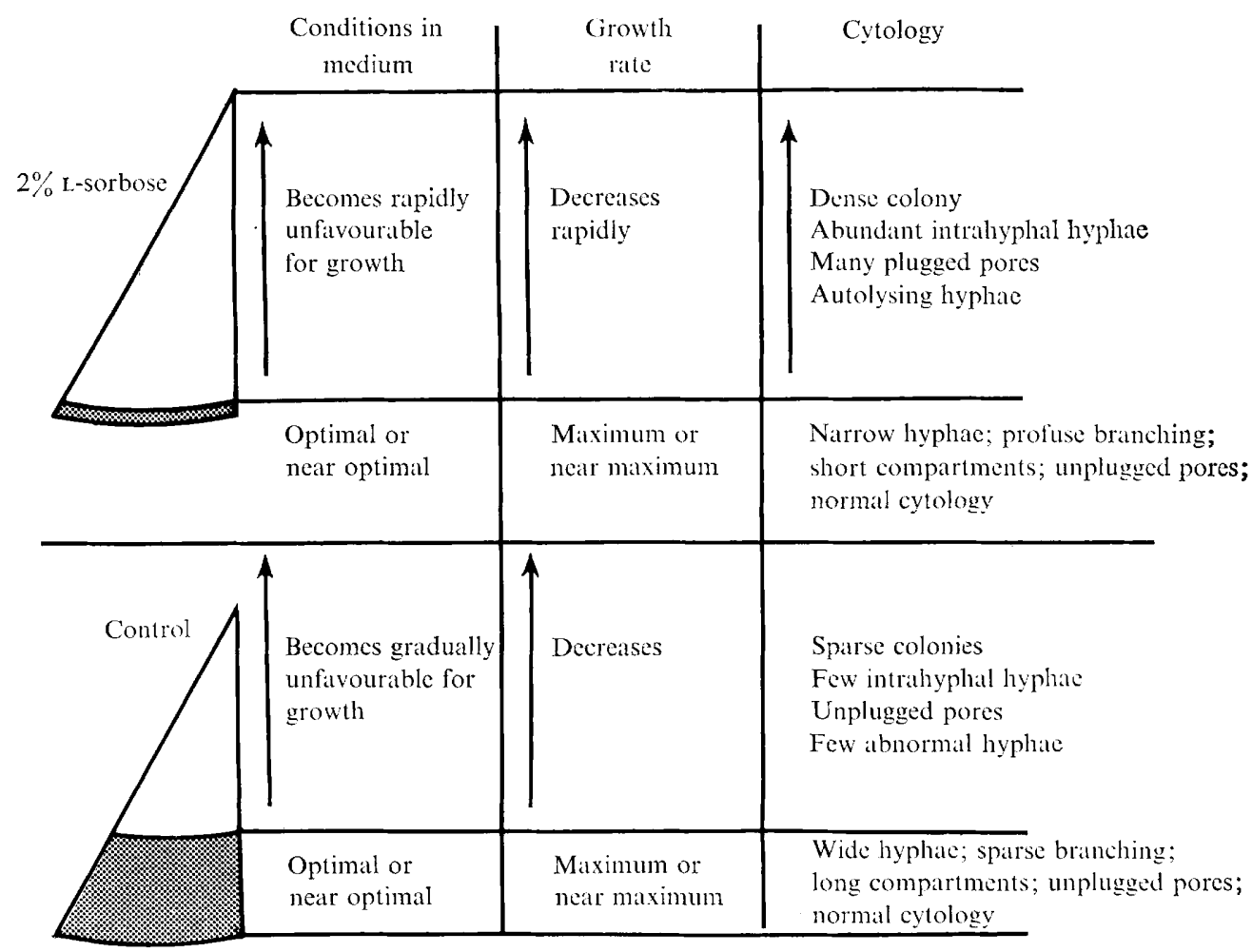

$=$ Peripheral growth zone

$\square=$ Zone not contributing to colony expansion

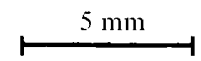

Fig. I2. Proposed changes in colonies of Neurospora crassa spco 9 caused by L-sorbose.

Arrows indicate direction of change. 
Table 4. Peripheral growth zones and theoretical and observed radial growth rates of colonies of Neurospora crassa spco 9 on Vogel's medium containing and lacking $2 \%$ L-sorbose

\begin{tabular}{|c|c|c|c|c|}
\hline Medium & $\begin{array}{l}\text { Width of } \\
\text { peripheral } \\
\text { growth zone } \\
\text { of colonies } \\
(w, \mu \mathrm{m})\end{array}$ & $\begin{array}{c}\text { Theoretical } \\
\text { colony } \\
\text { radial } \\
\text { growth rate* } \\
\left(K_{r t}, \mu \mathrm{m} / \mathrm{h}\right)\end{array}$ & $\begin{array}{c}\text { Observed } \\
\text { colony } \\
\text { radial } \\
\text { growth rate } \\
\left(K_{r}, \mu \mathrm{m} / \mathrm{h}\right)\end{array}$ & $\frac{K_{r}}{K_{r t}} \times 100$ \\
\hline Vogel's & $3930 \dagger$ & II9I & I 133 & 96 \\
\hline Vogel's $+2 \%(w / v)$ L-sorbose & 525 & 158 & 100 & 63 \\
\hline
\end{tabular}

* Calculated from, $K_{r l}=\alpha w$, where $w=$ width of the colony's peripheral growth zone and $\alpha=$ organism's specific growth rate (Table 2 ).

$\dagger$ From Trinci, $1973 a$.

The relationship between the length and the extension rate of severed peripheral hyphae of colonies of spco 9 grown on $2 \%$ L-sorbose is shown in Fig. II. The width of the peripheral growth zone of these colonies was thus reduced from about $4 \mathrm{~mm}$ in the absence of L-sorbose (Trinci, I973a) to about $0.5 \mathrm{~mm}$ in its presence (Table 4). The theoretical radial growth rates $\left(K_{r t}\right)$ of colonies of spco 9 grown in the presence and absence of L-sorbose were calculated from equation (I) using the organism's specific growth rate in batch culture (Table 2) and the width of the peripheral growth zone of their colonies (Table 4). Table 4 clearly shows that the reduction in the radial growth rate of colonies grown on media containing L-sorbose could be largely correlated with the observed decrease in the width of the colony's peripheral growth zone.

The proposed features of colonies of spco 9 grown in the presence and absence of $\mathrm{L}-$ sorbose are summarized in Fig. $\mathbf{2}$.

\section{DISCUSSION}

The results (their Fig. I) of Crocken \& Tatum (I968) support the view that L-sorbose has only a slight effect on the maximum specific growth rate of Neurospora crassa. Even on solid medium, the initial rate of hyphal growth is only slightly reduced by L-sorbose (Table 2). The observed decrease in growth rate in the presence of L-sorbose may possibly be caused by a reduction in the water activity of the medium. The most significant affect of $\mathrm{L}$-sorbose is that it induces an increase in branch initiation on solid medium; it thus effects the spatial distribution of hyphae, but has little influence on their maximum growth rate. The dense colonies formed on media containing L-sorbose probably have narrower peripheral growth zones than control colonies because the rate at which adverse conditions (e.g. nutrient or oxygen limitation, $\mathrm{pH}$ changes, accumulation of products of secondary metabolism) are established in the medium is related to hyphal density; adverse conditions are established closer to the margin of dense than of sparse colonies. This conclusion is supported by the cytological observations made in the present study. In batch culture the changes (e.g. oxygen tension, $\mathrm{pH}$, chemical) which result from the organism's growth are averaged throughout the entire medium.

There is a correlation between the width of the peripheral growth zone of colonies of spco 9 (Table 4) and the distance from the margin of the colony at which septal pores are plugged (Fig. 7) and autolysis occurs. However, we consider it unlikely that septal plugging is the primary event which regulates the width of the peripheral growth zone (Trinci \& Collinge, 1973). There are probably direct relationships between the lengths of the peripheral growth zones of hyphae and their diameters and compartment lengths. 
It has been suggested that synthesis of wall glucan in yeasts occurs by insertion of glucose into glucan chains at cuts made by extracellular glucanases; 2-deoxyglucose is thought to inhibit the activity of glucan synthetases and in so doing allows an accumulation of breaks in glucan polymers and causes wall lysis (Johnson, I968 $a$ and I968 b). L-Sorbose inhibits the activity (in vivo and in vitro) of $\beta \mathrm{I}-3$ glucan synthetase (Mishra \& Tatum, I972) and causes a decrease in the $\beta \mathrm{I}-3$ glucan content of Neurospora crassa walls (Mahadevan \& Tatum, 1965; Crocken \& Tatum, 1968). Thus in $N$. crassa L-sorbose may, like 2-deoxyglucose in yeasts, upset the balance between synthesis and cleavage of glucan polymers with the result that wall softening occurs and consequently the frequency of branch initiation is increased. This hypothesis is supported by the observation (Moore \& Stewart, 1972) that high concentrations of 2-deoxyglucose cause lysis of the hyphal walls of Coprinus lagopus. It is not known why L-sorbose should not have a similar morphogenetic effect on hyphae grown in submerged culture.

The results of Cirillo (I962) and Van Uden (1967) on the other hand suggest that L-sorbose competitively inhibits the uptake (both active and facilitated diffusion) of glucose by Saccharomyces cerevisiae.

L-Sorbose may not be unique in having an inhibitory effect on the growth of moulds on solid medium but not in batch culture. Wilson \& Niederpruem (1967) found that although cellobiose induced Schizophyllum commune to grow in a 'colonial' form (dense, slow growing colonies) on solid medium it had no affect on the yield of the organism in batch culture. Further, Lysek \& Esser (197I) found that although galactose and L-sorbose inhibited the growth of Podospora anserina on solid medium they did not affect the uptake of glucose or fructose by the mould in batch culture. Any substance which increases the frequency of hyphal branching is likely to have the same effect on colony radial growth rate as L-sorbose.

\section{REFERENCES}

Caldwell, I. Y. \& Trinci, A. P. J. (I973). The growth unit of the mould Geotrichum candidum. Archiv fïr Mikrobiologie 88, I-IO.

CiRILlo, V. P. (I962). Mechanism of glucose transport across the yeast cell membrane. Journal of Bacteriology 84, 485-49r.

Clutterbuck, A. J. \& Roper, J. A. (1966). A direct determination of nuclear distribution in heterokaryons of Aspergillus nidulans. Genetical Research, Cambridge 7, 185-194.

Cochrane (1958). Physiology of Fungi. London: Chapman and Hall.

Crocken, B. \& TAtum, E. L. (I968). The effect of sorbose on metabolism and morphology of Neurospora. Biochemica et biophysica acta $\mathbf{5} \mathbf{6}, \mathrm{I}-8$.

Johnson, B. F. (1968a). Dissolution of yeast glucan induced by 2-deoxyglucose. Experimental Cell Research 50, 692-694.

Johnson, B. F. (1968b). Lysis of cell walls induced by 2-deoxyglucose at their sites of glucan synthesis. Journal of Bacteriology 95, I I 69-1 I 72.

LHOAS, P. (I968). Growth rate and haploidization of Aspergillis niger on medium containing p-fluorophenylalamine. Genetical Research, Cambridge 12, 305-315.

LySEK, G. \& ESSER, K. (197I). Rhythmic mycelial growth in Podospora anserina. II. Evidence for a correlation with carbohydrate metabolism. Archiv fiir Mikrobiologie 75, 360-373.

Mahadevan, P. R. \& Tatum, E. L. (1965). Relationship of the major constituents of the Neurospora crassa cell wall to wild type and colonial morphology. Journal of Bacteriology 90, 1073-108 r .

Mishra, N. C. \& TATUM, E. L. (1972). Effect of L-sorbose on polysaccharide synthetases of Neurospora crassa. Proceedings of the National Academy of Sciences of the United States of America 69, 313-317.

Moore, D. \& StewarT, G. R. (I 972). Effects of 2-deoxy-D-glucose, D-glucosamine and L-sorbose on the growth of Coprinus lagopus. Journal of General Microbiology 7r, 333-342.

Righelato, R. C., Trinci, A. P. J., Pirt, S. J. \& Peat, A. (i968). The influence of maintenance energy and growth rate on the metabolic activity, morphology and conidiation r,i Penicillium chrysogenum. Journal of General Microbiology 50, 399-4I 2. 
Tatum, E. L., Barratt, R. W. \& Cutter, V. M. (I949). Chemical induction of colonial paramorphs in Neurospora and Syncephalastrum. Science, New York I09, 509-5I I.

TRINCI, A. P. J. (I97I). Influence of the width of the peripheral growth zone on the radial growth rate of fungal colonies on solid media. Journal of General Microbiology 67, 325-344.

TrINCI, A. P. J. (1973a). Growth of wild type and spreading colonial mutants of Neurospora crassa in batch culture and on agar medium. Archiv für Mikrobiologie (in the Press).

TrincI, A. P. J. (1973b). The hyphal growth unit of wild type and spreading colonial mutants of Neurospora crassa. Archiv für Mikrobiologie (in the Press).

Trinci, A. P. J. \& Collinge, A. (1973). Structure and plugging of septa of wild type and spreading colonia mutants of Neurospora crassa. Archiv für Mikrobiologie (in the Press).

Trinci, A. P. J. \& Righelato, R. C. (197I). Changes in constituents and ultrastructure of hyphal compartments during autolysis of glucose starved Penicillium chrysogenum. Journal of General Microbiology 6o, 234-249.

VAN UdEN, N. (1967). Transport-limited fermentation and growth of Saccharomyces cerevisiae and its competitive inhibition. Archiv für Mikrobiologie 58, I 55-168.

Vogel, H. J. (1956). A convenient growth medium for Neurospora (medium N). Microbiology and Geneticat Bulletin $\mathbf{3}, 42$.

Wilson, R. W. \& NeIderpruem, D. J. (I967). Cellobiose as a paramorphogen in Schizophyllum commune. Canadian Journal of Microbiology 16, 629-634. 